\title{
Ritonavir Exhibits Limited Efficacy as a Single Agent in Treating Aggressive Mantle Cell Lymphoma
}

Tara M. Nordgren, Ganapati V. Hegde and Shantaram S. Joshi*

Department of Genetics, Cell Biology and Anatomy, University of Nebraska Medical Center. Omaha, NE 68198-6395, USA

\begin{abstract}
Background: Mantle Cell Lymphoma (MCL) is an aggressive B cell malignancy accounting for $6 \%$ of nonHodgkin's lymphoma cases in the US. While various therapies are available to treat MCL, patients relapse within 3 to 4 years following treatment from therapy-resistant $\mathrm{MCL}$, making $\mathrm{MCL}$ carry one of the worst prognoses of all nonHodgkin's B cell lymphomas. A better understanding of the biological mechanisms of relapse and therapy-resistance in $\mathrm{MCL}$ is vital for developing mechanisms to target relapsing $\mathrm{MCL}$, and providing better care for patients. Recent studies implicate the NFKB pathway and survivin in promotion of aggressive, therapy-resistant MCL. Therefore, we tested the efficacy of inhibiting this pathway in three MCL lines (GP, recently-developed GRL, and JVM2) using the protease inhibitor ritonavir (Abbott Laboratories), which has been shown to downregulate NFKB targets, including survivin, in other hematological malignancies.
\end{abstract}

Methods: MCL cells were incubated with ritonavir then assessed for changes in proliferation, apoptosis, and activation of NFKB transcriptional targets. In addition, in vivo studies were performed to assess ritonavir's utility as a single agent in MCL treatment using an immune-deficient mouse model of human MCL.

Results: When MCL cell lines were incubated with ritonavir in vitro, they exhibited reduced proliferation, increased apoptosis, and downregulation of NFKB pathway targets. However, no effect was seen when testing ritonavir as a single agent in vivo. Although, treatment with ritonavir plus vincristine in vitro revealed significant reduction in the proliferation of MCL compared to either treatment alone.

Conclusions: These studies suggest ritonavir is not suitable as a single-agent therapy for MCL. However, studies combining ritonavir plus vincristine in vitro suggest ritonavir may be effective in multi-pronged treatment approaches for MCL. These findings necessitate further studies to determine ritonavir's utility within a multi-pronged treatment approach for treating therapy-resistant MCL.

Keywords: Mantle cell lymphoma; Ritonavir; Refractory lymphoma; Protease inhibitors; NFkB; Survivin

\section{Introduction}

Mantle Cell Lymphoma (MCL) is an aggressive non-Hodgkin's B cell lymphoma. MCL is characterized by $\mathrm{CD}^{+} \mathrm{CD} 23^{-}$monoclonal B cells containing a t $(11 ; 14)$ (q13; q32) translocation causing overexpression of cyclinD1, a protein involved in cell proliferation [1]. Accounting for about $6 \%$ of all non-Hodgkin's lymphomas, MCL has multiple treatment options, including several chemotherapy regimens with and without rituximab, radio-immunotherapies, small molecule inhibitors, and stem cell transplantation. While these treatments are reasonably effective in lowering tumor burden in patients [2], relapse ultimately occurs due to the failure of these treatments to eliminate residual tumor cells. Consequently, even with current therapeutic options, median patient survival with MCL is only 3 to 4 years. MCL therefore carries the worst survival prognosis of all B cell lymphomas [3]. New treatment regimens are needed to not only target primary tumor burden, but to specifically target residual tumor, thus offering a greater survival benefit to patients.

Recent studies have suggested that aberrant activation of the NFKB pathway including upstream signaling modulators PI3K and Akt may be contributing to the aggressive nature of MCL [4-7]. These signaling patterns, which are broadly involved in cell growth and proliferation [8], have been shown to be constitutively active in MCL, leading to a further upregulation of certain cell cycle-related proteins, including MCL's characteristic driver of malignancy, cyclin D1 [9]. Inhibition of key molecules in this pathway through the use of NFKB pathway inhibitors has shown promise in lowering cyclin D1 expression, leading to a decrease in MCL proliferation [9]. These findings make the NFkB pathway an interesting target for combined therapy designs.

Overexpression of survivin-a target molecule in the NFKB pathway-has been implicated in promoting the proliferation an apoptosis-resistance of MCL [1]. Survivin, which is a member of the inhibitor-of-apoptosis protein family, is involved in preventing apoptosis by directly binding and inhibiting the executioner caspases 3 and 7 [10]. Additionally, survivin plays a role in cell cycle regulation by associating with proteins in the mitotic spindle apparatus [11]. Survivin is not expressed in most adult tissue but is commonly overexpressed in malignancies, making it a unique tumor-specific marker and antigen [12]. Therefore, the use of NFKB/survivin-targeting therapeutics may prove to be successful inhibitors of survivin, thus sensitizing MCL to more conventional therapeutics [11-15]. Other NFkB transcriptional targets also have roles in proliferation and survival that may be

*Corresponding author: Shantaram S. Joshi, PhD, Department of Genetics, Cell Biology and Anatomy, 986395 University of Nebraska Medical Center Omaha, NE 68198-6395, USA, Tel: (402) 559-4165; Fax: (402) 559-3400; E-mail: ssjoshi@unmc.edu

Received March 12, 2012; Accepted April 09, 2012; Published April 11, 2012

Citation: Nordgren TM, Hegde GV, Joshi SS (2012) Ritonavir Exhibits Limited Efficacy as a Single Agent in Treating Aggressive Mantle Cell Lymphoma. J Cancer Sci Ther 4: 061-068. doi:10.4172/1948-5956.1000112

Copyright: (C) 2012 Nordgren TM, et al. This is an open-access article distributed under the terms of the Creative Commons Attribution License, which permits unrestricted use, distribution, and reproduction in any medium, provided the original author and source are credited. 
relevant to the progression, therapy-resistance, and/or aggressiveness of MCL. These include the anti-apoptotic protein $\mathrm{Bcl} 2$ and the cell cycle regulator Cyclin D2 $[8,16,17]$. Therefore, targeting the NFkB pathway in MCL may significantly impair the progression of this aggressive disease.

Recent reports have shown the FDA-approved protease inhibitor ritonavir (Abbott Laboratories) may be able to interact and abrogate signaling through the NFKB pathway, resulting in downregulation of survivin and cyclin $\mathrm{D} 2$, and alteration of NFKB binding abilities. These effects result in decreased growth, induction of apoptosis, and/or increased survival when ritonavir is tested against both hematological and non-hematological malignancies, including breast cancer, prostate cancer, lung adenocarcinoma, lymphoblastoid $\mathrm{B}$ cells, and $\mathrm{T}$ cell leukemia [14,18-21]. Therapies that incorporate targeting of survivin in aggressive non-Hodgkin's lymphoma have shown some therapeutic promise when combined with conventional combinatorial treatments in vitro and in vivo due to their multiple-target approaches $[13,22]$. Furthermore, mounting evidence confirms the necessity for multipronged, multiple target treatment approaches in the treatment of MCL [23,24]. Recent reports have shown ritonavir in combination with other therapies may enhance certain cancer treatments, including extra-nodal marginal zone B cell lymphoma (mucosal-associated lymphoid tissue type), human sarcoma, and various solid tumors, along with enhancing Human Immunodeficiency Virus (HIV) treatments for which it was originally designed [25-28]. Thus, the potential for ritonavir as a treatment for MCL may be promising.

Therefore, we chose to target the NFKB pathway and its targets, including survivin, using ritonavir to assess the potential of this therapy in inhibiting the proliferation and survival of MCL. We utilized three MCL cell lines: JVM2, Granta-519 (GP), and a therapy-resistant MCL cell line, GRL [29], which was derived from GP and represents relapsing MCL and represents highly-aggressive MCL. Briefly, we tested the ability of ritonavir alone or in combination with vincristine to inhibit proliferation and induce apoptosis in the MCL cell lines. Alteration of the NFkB transcriptional targets BCL2, CCND2 (cyclin D2), and BIRC5 (survivin) following ritonavir exposure was also assessed, and the protein levels and localization of the NFKB subunit p65 was analyzed. In addition, in vivo studies were performed to assess ritonavir's utility as a single agent in treating MCL. We found that the use of ritonavir in treating MCL as a single agent has little efficacy. However, further studies are warranted to test ritonavir's efficacy when used in a multi-pronged treatment approach.

\section{Materials and Methods}

\section{Cell culture}

The MCL cell lines, Granta 519 and JVM2, were purchased from Deutsche Sammlung von Mikroorganismen und Zellkulturen (DSMZ, Germany). The derivative cell line, GRL was developed from Granta 519, as described below. These cell lines were maintained in DMEM media (Invitrogen, CA) containing 10\% fetal bovine serum (FBS) (U.S. Bio-Technologies Inc., PA), 1X penicillin/streptomycin (Invitrogen, $\mathrm{CA}$ ) and $1 \mathrm{X}$ L-Glutamine (Invitrogen, CA), termed DF-10.

\section{Isolation of therapy-resistant cell line GRL from NOD/ SCID mice inoculated with GP and treated with CHOP + bortezomib therapy}

The GRL cell line was developed as previously described [29]. Briefly, at Day 0 of the study, NOD/SCID mice were irradiated with 2.15 gray irradiation using the cobalt-60 source at the UNMC Experimental Irradiation Facility. On Day 1, mice were inoculated via tail vein injection with $2 \times 10^{6} \mathrm{GP}$ cells suspended in $100 \mu \mathrm{l}$ sterile media. On Day 11, mice were given three doses administered at every-other day intervals consisting of $30 \mathrm{mg} / \mathrm{kg}$ cyclophosphamide (Sigma-Aldrich, MO) (via intravenous injection), $0.375 \mathrm{mg} / \mathrm{kg}$ vincristine (SigmaAldrich, MO) (via intravenous injection), $2.5 \mathrm{mg} / \mathrm{kg}$ doxorubicin (Sigma-Aldrich, MO) (via intravenous injection), and $0.15 \mathrm{mg} / \mathrm{kg}$ prednisone (Sigma-Aldrich, MO) (given orally). On Day 24, mice were administered four doses of $0.5 \mathrm{mg} / \mathrm{kg}$ bortezomib given intravenously on every-other day intervals. When mice became moribund following treatment regimen (evidenced by weight loss, hunching back, ruffled fur, excessive dehydration, and/or hind-limb paralysis), mice were euthanized via $\mathrm{CO}_{2}$ chamber method. Livers were harvested from mice and minced into single cell suspensions. Cells were washed at least three times in DMEM media, then cultured in DF10 in 5\% $\mathrm{CO}_{2}$ at $37^{\circ} \mathrm{C}$ until murine cells in cultures were eliminated and pure cultures of human tumor cells were obtained. If necessary, cultures were treated with $50 \mu \mathrm{g} / \mathrm{ml}$ gentamycin (Sigma-Aldrich, MO) to avoid/eliminate contamination.

\section{In vitro growth assay}

Ten thousand GP, GRL1, and JVM2 cells were cultured in 96well plates in $200 \mu \mathrm{l}$ DF10 medium, and the growth of these cells were determined at 24, 48 and 72 hours by MTT assays. Briefly, $25 \mu \mathrm{l}$ of MTT reagent (Sigma-Aldrich, MO) ( $5 \mathrm{mg} / \mathrm{ml}$ in PBS) was added to the culture and incubated for 2 hours before the respective time point, and the cells were lysed using an SDS-based reagent. The intensity of the color developed was determined at $570 \mathrm{~nm}$ using a plate reader. To determine the effects of ritonavir alone, or in combination with vincristine on the GP, GRL, and JVM2 cell lines, ten thousand cells were cultured in the presence of $10 \mu \mathrm{M}$ ritonavir (Abbott Laboratories, IL), $25 \mathrm{nM}$ doxorubicin, and/or $5 \mu \mathrm{M}$ vincristine for 24,48 , or 72 hours. The growth rate was measured by MTT and assays as explained above. The change of growth of treated MCL cells was determined relative to untreated cells. Statistical significance was determined using student's $\mathrm{T}$ test analyses.

\section{Real-time PCR}

RNA was isolated from fresh passages or following treatment with ritonavir of GP, GRL and JVM2 cells using Trizol reagent (Invitrogen, CA). cDNA was synthesized from RNA, and real-time PCR was performed for transcripts associated with the NFKB signaling pathway using an ABI PRISM 7000 Sequence Detection System, as described previously [30]. Statistical significance was determined using student's T- test analyses. Primers used in these experiments are as follows:

BIRC5: F: 5'-ACCTGAAGCTTCCTCGACA-3', R: 5'-AACCCTTCCCAGACTCCACT-3'; BCL2: F: 5'- GCATGCGGCCTCTGTTTGATTTCT-3', R: 5'-AGGCATGTTGACTTCACTTGTGGC-3'; CCND2: F: 5'-CTTCGCTTCTGGTATCTGGC-3', R: 5'-TGAGGAATGTTGTGATGGG-3'; HPRT: F: 5'-AGGGTGTTATTCCTCATGGAC-3'， R: 5'-GTAATCCAGCAGGTCAGCAAAG-3'.

\section{Apoptosis assay}

GP, GRL, and JVM2 cells were cultured at a concentration of $1 \times 10^{6}$ cells $/ \mathrm{ml}$ in $10 \mu \mathrm{M}, 20 \mu \mathrm{M}$, or $40 \mu \mathrm{M}$ ritonavir for 24,48 , or 72 hours. The amount of the cell population undergoing apoptosis was then assessed using the AnnexinV:FITC apoptosis assay kit (BD Pharmingen, CA), 
performed according to manufacturer's instructions. Flow cytometry was performed in the flow cytometry core facilities of the University of Nebraska Medical Center, Omaha, NE. Statistical significance was determined using student's $\mathrm{T}$ test analyses.

\section{Immuno-cytochemistry}

Immunocytochemistry for the $\mathrm{NF \kappa B}$ subunit $\mathrm{p} 65$ was performed to assess changes in protein level expression and localization at 24 hours following $10 \mu \mathrm{M}$ ritonavir treatment in the GP, GRL, and JVM2 cell lines. Briefly, cells were adhered to poly-L-lysine-coated slides using a Shandon Cytospin III. Cells were fixed in paraformaldehyde, rinsed in Phosphate-buffered Saline (PBS), and then permeabilized with a Triton-X solution. Cells were washed, then blocked in $1 \%$ BSA in PBS, then incubated with primary NFkB p65 antibody (Santa Cruz Biotech, CA) at a 1:100 dilution. Cells were washed and incubated in goat $\alpha$-mouse IgG-HRP antibody (Santa Cruz Biotech, CA) at a 1:1000 dilution. The VectaShield DAB kit (Vector Labs, CA) was used to develop slides, which were then cover-slipped and viewed via light microscopy.

\section{Western blotting}

Western blotting for the NFKB subunit p65 was performed to assess changes in protein level expression at 24 hours following $10 \mu \mathrm{M}$ ritonavir treatment in GP, GRL, and JVM2 cell lines. Protein was isolated from cells using Mammalian Protein Extraction Reagent (Thermo Fisher Scientific, IL). Proteins were run on 4-20\% Ready Gels (Bio-rad, CA) and transferred onto PVDF membranes. Membranes were probed with mouse a-human p65 antibody (Santa Cruz Biotechnology, Inc, CA) and mouse $\alpha$-human actin antibody (Novus Biologicals, CO) at 1:400 dilutions. Secondary antibody utilized was goat $\alpha$-mouse IgG-HRP antibody (Santa Cruz Biotech, CA) at a 1:1000 dilution. VectaShield DAB kit was used to develop the membrane, which was then imaged using a PowerShot S X 200 camera.

\section{In vivo studies}

For in vivo studies, 6-8 week old NOD.CB17-Prkdc scid/J mice were purchased from (Jackson Laboratories, ME) and housed in the UNMC Comparative Medicine Animal Facilities.

\section{Studies assessing efficacy of ritonavir in NOD/SCID mice inoculated with GP}

On Day 0 of the studies, NOD/SCID mice were irradiated with 2.15 gray using the UNMC cobalt irradiator facility. On Day 1, mice were inoculated with $1 \times 10^{6} \mathrm{GP}$ cells suspended in $100 \mu \mathrm{l}$ sterile media by tail vein injection. In one study, mice were then treated with 7 consecutive daily doses of $30 \mathrm{mg} / \mathrm{kg}$ ritonavir (administered orally, suspended in $50 \mu$ milk vehicle) beginning on Day 11 . This dosing is consistent with that used in previous studies $[14,20]$. In a second study, mice were treated with 30 consecutive daily doses of $30 \mathrm{mg} / \mathrm{kg}$ ritonavir beginning on Day 8. For ritonavir treatment, $30 \mathrm{mg} / \mathrm{kg}$ doses were given for 30 consecutive days. Mice were monitored for survival. When mice became moribund (as evidenced by weight loss, hunching back, ruffled fur, excessive dehydration, and/or hind-limb paralysis), mice were euthanized using the $\mathrm{CO}_{2}$ chamber method. Statistical significance was determined using Kaplan-Meier Log-Rank test analyses.

\section{Results}

\section{Effects of ritonavir on the proliferation of MCL cell lines in vitro}

In order to be an effective treatment for MCL, ritonavir must inhibit the proliferation of this aggressive malignancy. Ritonavir has previously shown success in the inhibition of proliferation of other cancer types [18-20]. In order to assess the ability of ritonavir to inhibit proliferation in vitro, the GP, GRL, and JVM2 cell lines were incubated with $10 \mu \mathrm{M}$ ritonavir for 24,48 , or 72 hours, and the proliferation of the cells was assessed using MTT assays. The $10 \mu \mathrm{M}$ ritonavir concentration was chosen as this is the calculated approximate concentration present in the serum of a patient on a daily regimen of ritonavir, as currently approved by the FDA. At all time points, the GP, GRL, and JVM2 cells treated with $10 \mu \mathrm{M}$ ritonavir exhibited significantly lower proliferation than control (untreated) cells, as shown in (Figure 1 Left Panel). This suggests ritonavir is successful in inhibiting the growth of aggressive, refractory MCL in vitro.

\section{Induction of apoptosis following ritonavir treatment in MCL cell lines}

Ritonavir has been previously shown to inhibit the proliferation and induce apoptosis in several malignancies, including hematologicallyrelevant adult $\mathrm{T}$ cell leukemia and lymphoblastoid $\mathrm{B}$ cells $[14,20]$. Therefore, to test the ability of ritonavir to induce apoptosis in MCL, AnnexinV:FITC apoptosis assays were performed. GP, GRL, and JVM2 cells were incubated with $10 \mu \mathrm{M}, 20 \mu \mathrm{M}$, and $40 \mu \mathrm{M}$ ritonavir for 48 and 72 hours and analyzed flow cytometrically for the binding of AnnexinV to indicate apoptosis induction. As shown in (Figure 1 Right Panel), treatment with ritonavir for 48 or 72 hours induces apoptosis in the GP, GRL, and JVM2 cell lines in a dose-dependent manner. This corroborates with previous studies exhibiting the ability of ritonavir to inhibit proliferation and induce apoptosis in malignant cells of hematopoietic lineage.

\section{Changes in transcript level of NFkB targets following ritonavir treatment}

Mechanistically, ritonavir has been shown to act on malignancies via modification of NFKB pathway activity [14, 18-19]. Therefore, to ascertain the mechanisms of proliferation inhibition and induction of apoptosis following ritonavir treatment in MCL, transcriptional changes to NFKB pathway targets were assessed. GP, GRL1, and JVM2 cell lines were treated with $10 \mu \mathrm{M}$ ritonavir for 6 hours then harvested for RNA. Real-time PCR was performed to assess the effects of ritonavir on transcript levels of selected NFKB targets relevant to MCL. Transcript levels of the pathway targets BIRC5 (survivin transcript), BCL2 (anti-apoptotic Bcl2 protein), and CCND2 (cyclin D2 transcript) were compared between treated versus untreated groups. As seen in (Figure 2), at 6 hours following $10 \mu \mathrm{M}$ ritonavir treatment, the transcript levels of BCL2 and CCND2 were significantly down-regulated in the treated cells as compared to untreated cells. Interestingly, the transcript levels of BIRC5 were significantly down-regulated in GP and GRL1 at 6 hours, but not in JVM2, suggesting this MCL line may have signaling interactions that differ from those of the Granta 519-type lines. Overall, the downregulation of the BCL2, CCND2, and BIRC5 (except in JVM2) transcripts in ritonavir-treated cells suggest ritonavir may be acting on or reducing the level of NFKB signaling in these treated cells.

\section{Alterations in protein levels of p65 following ritonavir treatment}

Due to implications of ritonavir acting through modulation of $\mathrm{NF \kappa B}$ signaling, the protein levels and localization of the NFkB subunit p65 were evaluated in GP, GRL, and JVM2 cell lines following 24 hours treatment with $10 \mu \mathrm{M}$ ritonavir. Figure 3 exhibits the protein levels of p65 seen in GP, GRL, and JVM2 at 24 hours following ritonavir 
Citation: Nordgren TM, Hegde GV, Joshi SS (2012) Ritonavir Exhibits Limited Efficacy as a Single Agent in Treating Aggressive Mantle Cell Lymphoma. J Cancer Sci Ther 4: 061-068. doi:10.4172/1948-5956.1000112
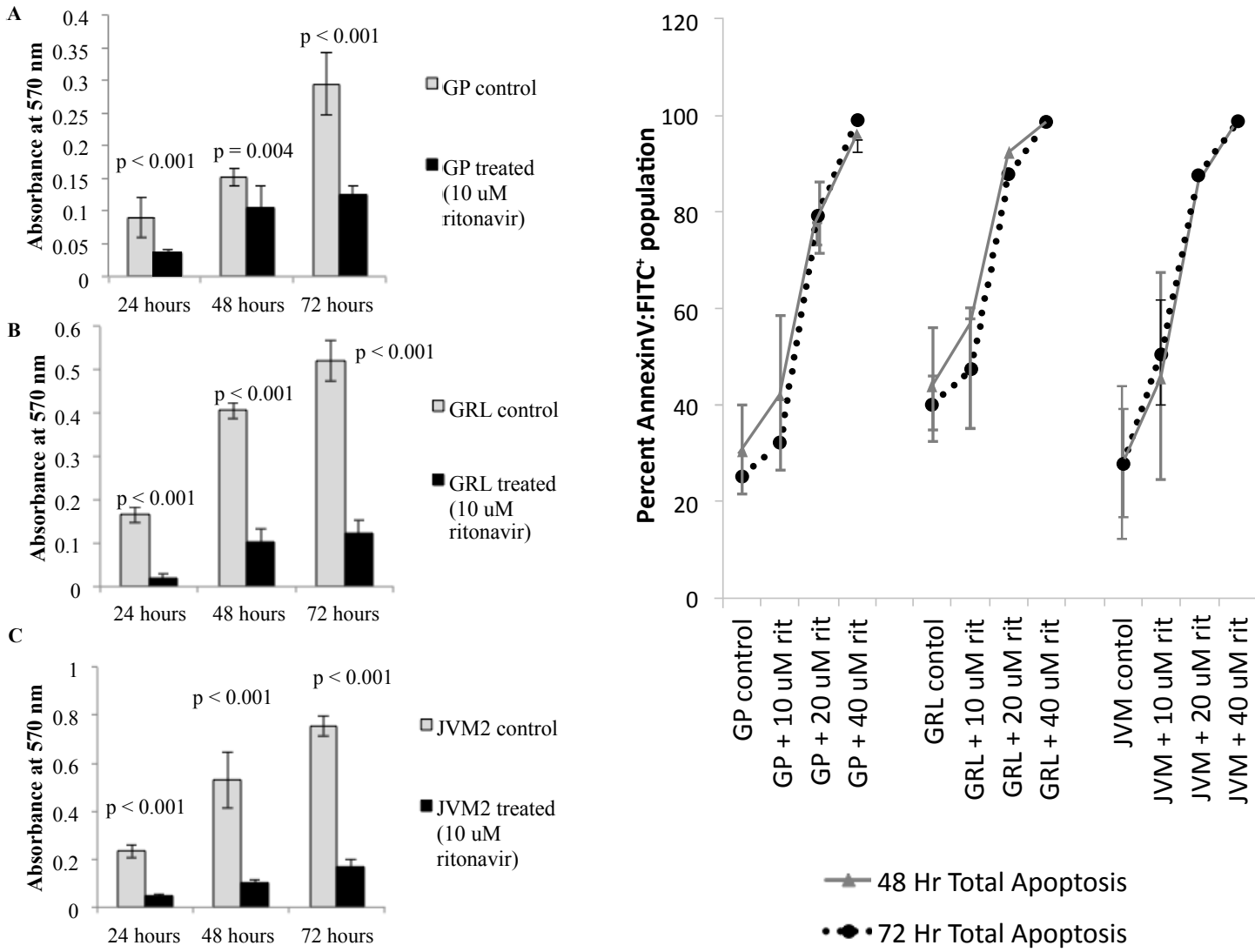

Figure 1: Effects of $10 \mu \mathrm{M}$ ritonavir treatment on the proliferation and induction of apoptosis in MCL. Left Panel: proliferation of GP (A), GRL (B), and JVM2 (C) after 24,48 , or 72 hour incubation periods with $10 \mu \mathrm{M}$ ritonavir, as assessed using MTT assays. GP, GRL, and JVM2 cell lines were seeded at 10,000 cells per well in a 96 well plate and incubated with $10 \mu \mathrm{M}$ ritonavir for 24,48 , or 72 hours. Two hours prior to the given time point, $5 \mathrm{mg} / \mathrm{ml}$ MTT was added to each well, and cells were harvested at time point using a detergent-based buffer. Proliferation of the cells was assessed calorimetrically using a spectrophotometer. Right Panel: Induction of apoptosis in GP, GRL, and JVM2 after 48 or 72 hour incubation periods with $10 \mu \mathrm{M}$ ritonavir, as assessed using AnnexinV:FITC apoptosis assay. GP, GRL, and JVM2 cells $\left(1 \times 10^{6}\right.$ cells) were treated with $10 \mu \mathrm{M}$ ritonavir for 48 or 72 hours. The AnnexinV:FITC substrate and/or propidium iodide was then added to cells, and cells were analyzed via flow cytometry for positive staining. Cells positive for AnnexinV alone, or both AnnexinV and propidium iodide considered to be apoptotic.

treatment as assessed by immuno-cytochemical staining and western blotting. While western blotting reveals no significant change in total p65 protein levels, the immunocytochemical staining suggests a modest downregulation in nuclear staining of p65 in the GP and GRL cells treated with ritonavir, as compared to control. When activated, p65 will translocate from the cytoplasm to the nucleus. Therefore, the reduction in nuclear staining seen following ritonavir treatment in GP and GRL cells suggests there may be inhibition of NFKB activity as a result of ritonavir treatment.

\section{Efficacy of seven day ritonavir treatment regimen in vivo in NOD/SCID mice inoculated with GP.}

In a preliminarily test of ritonavir's efficacy in vivo against the aggressive human MCL line GP, 10 immune-compromised NOD/ SCID mice were inoculated with $1 \times 10^{6} \mathrm{GP}$ cells via tail vein injection. Ten days following inoculation, 5 mice were given vehicle only, while 5 mice received $30 \mathrm{mg} / \mathrm{kg} /$ day ritonavir for 7 consecutive days. Mice were then observed for survival. Shown in (Figure 4 Left Panel), treatment with ritonavir did not significantly improve overall survival with this treatment regimen, suggesting a longer treatment regimen or combined treatment approach may be necessary for treating MCL with ritonavir in vivo.

\section{Efficacy of 30 day ritonavir treatment regimen in vivo in NOD/SCID mice inoculated with GP}

In determining the efficacy of a longer treatment course similar to those previously shown effective against lymphoblastoid $\mathrm{B}$ cells in vivo [14], 20 NOD/SCID mice were first inoculated with $1 \times 10^{6} \mathrm{GP}$ cells via tail vein injection. Seven days following the MCL injections, 10 mice were treated with vehicle only, while 10 mice were treated with $30 \mathrm{mg} /$ $\mathrm{kg}$ /day ritonavir for 30 consecutive days. Mice were then observed for survival. As shown in (Figure 4 Right Panel), treatment with ritonavir at this dosing regimen and schedule did not significantly improve overall survival as assessed using Kaplan-Meier Log Rank Test analyses ( $p=0.17$ ), thus suggesting a combined regimen may be necessary for treating MCL with ritonavir in vivo.

\section{Effects of ritonavir treatment in combination with vincristine treatment on the proliferation of MCL cell lines}

In the treatment of MCL, multi-pronged approaches incorporating targeting of multiple mechanisms of growth and proliferation have shown greater potential than single treatment therapies [23-24]. In addition, ritonavir treatment has been shown to be particularly effective in enhancing the efficacy of standard treatments in certain cancers $[18,26]$. Therefore, GP, GRL, and JVM2 cells were treated 

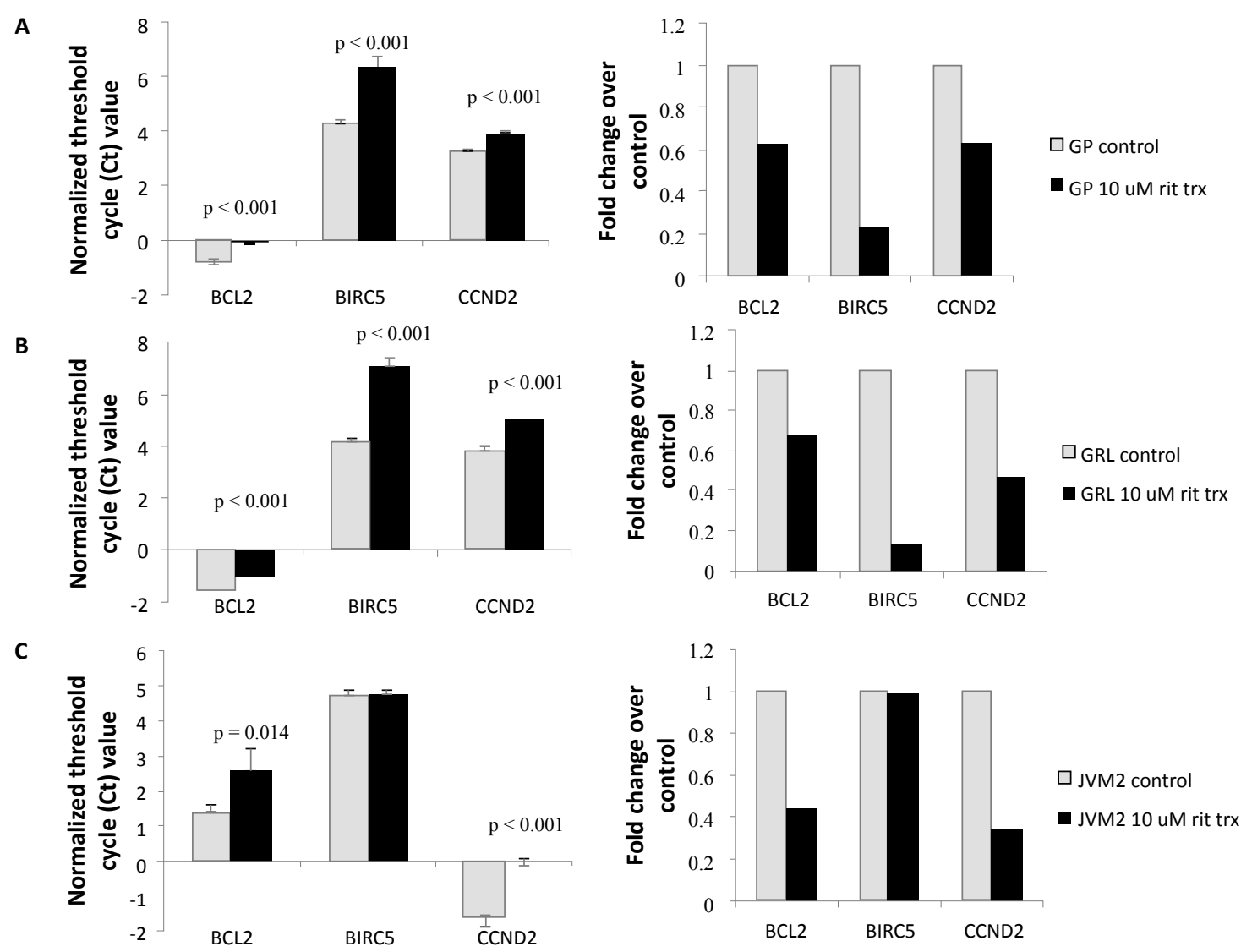

Figure 2: Effects of ritonavir treatment on NFKB target transcripts in MCL. Effects of 6 hours treatment with $10 \mu \mathrm{M}$ ritonavir on the mRNA expression levels of NFKB targets BCL2, BIRC5 (survivin), and CCND2 (cyclin D2) in GP (A), GRL (B), and JVM2 (C), as assessed using real time PCR. GP, GRL, and JVM2 cells (1 x $10^{6} \mathrm{cells}$ ) were treated with $10 \mu \mathrm{M}$ ritonavir for 6 hours. Cells were then spun down and RNA isolated via Trizol. RNA was used for subsequent cDNA preparation and transcript-level analyses. Graphs on the left show threshold cycle $(\mathrm{Ct})$ values of transcripts, normalized to the housekeeping control gene transcript HPRT. Graphs on the right show the correlative fold changes between untreated and treated cells.

with $10 \mu \mathrm{M}$ ritonavir and/or $5 \mu \mathrm{M}$ vincristine for 24,48 , or 72 hours. Vincristine, which is a vinca alkaloid and mitotic inhibitor, was utilized as it is represented in the standard MCL therapy of cyclophosphamide, vincristine, doxorubicin, and prednisone (CHOP), as well as other often-used clinical treatment regimens [24,31]. It is therefore plausible that targeting different mechanisms of proliferation and survival in MCL through combining the effects of ritonavir plus vincristine may lead to improved efficacies. Changes in proliferation of GP, GRL, and JVM2 cells at 24, 48, and 72 hours following ritonavir and/or vincristine treatments were assessed using MTT assays. As shown in (Figure 5), treating JVM2, GRL, or GP cells with a combination of vincristine and ritonavir resulted in a significant reduction of cell proliferation compared to vincristine alone or ritonavir alone (ritonavir alone to combined treatment: $\mathrm{p}<0.0041$ in GP, $\mathrm{p}<0.0095$ in GRL1, and $\mathrm{p}<$ 0.001 in JVM2 across all time intervals). This suggests ritonavir may have utility in MCL treatment as a partner in combined therapeutic regimens.

\section{Discussion}

The treatment of MCL represents an extremely difficult task for clinicians. While MCL patients often respond well to primary therapy, their period of remission is short-lived and gives way to aggressive disease that is nearly always resistant to currently available therapeutics. This issue presents an immediate and vital need for new therapeutic

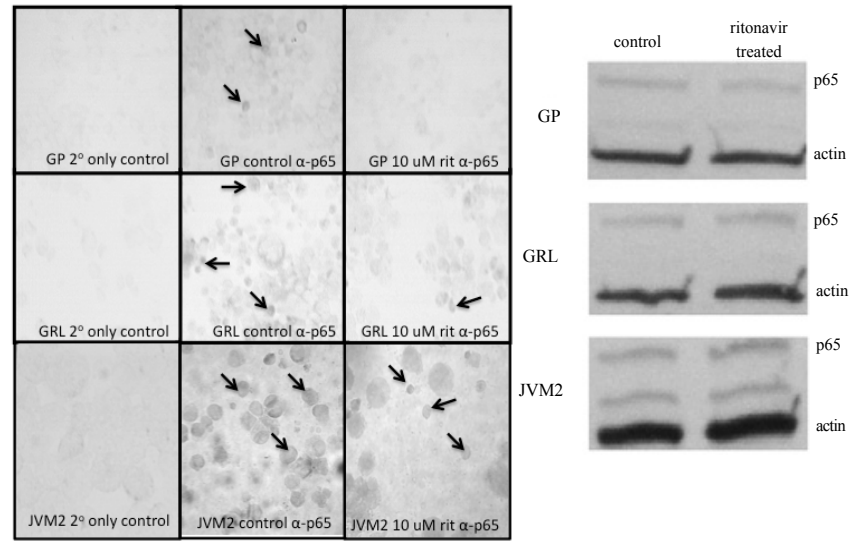

Figure 3: Effects of ritonavir treatment NFKB protein levels in MCL. Effects of 24 hours treatment with $10 \mu \mathrm{M}$ ritonavir on the protein level expression and localization of NFKB subunit p65 in GP, GRL, and JVM2 cell lines, as assessed using immunocytochemistry (left panel, 40X magnification) and western blotting (right panel). GP, GRL, and JVM2 cells $\left(1 \times 10^{6}\right.$ cells) were treated for 24 hours with $10 \mu \mathrm{M}$ ritonavir. For immunocytochemistry, cytospins were prepared and staining performed for $\mathrm{p} 65$. For western blotting, protein was then isolated with cell lysis buffer, run on SDS-PAGE gels, transferred to PVDF membranes, and blotted for p65. Arrows on immunohistochemistry figure indicate representative nuclear staining of $\mathrm{p} 65$. 

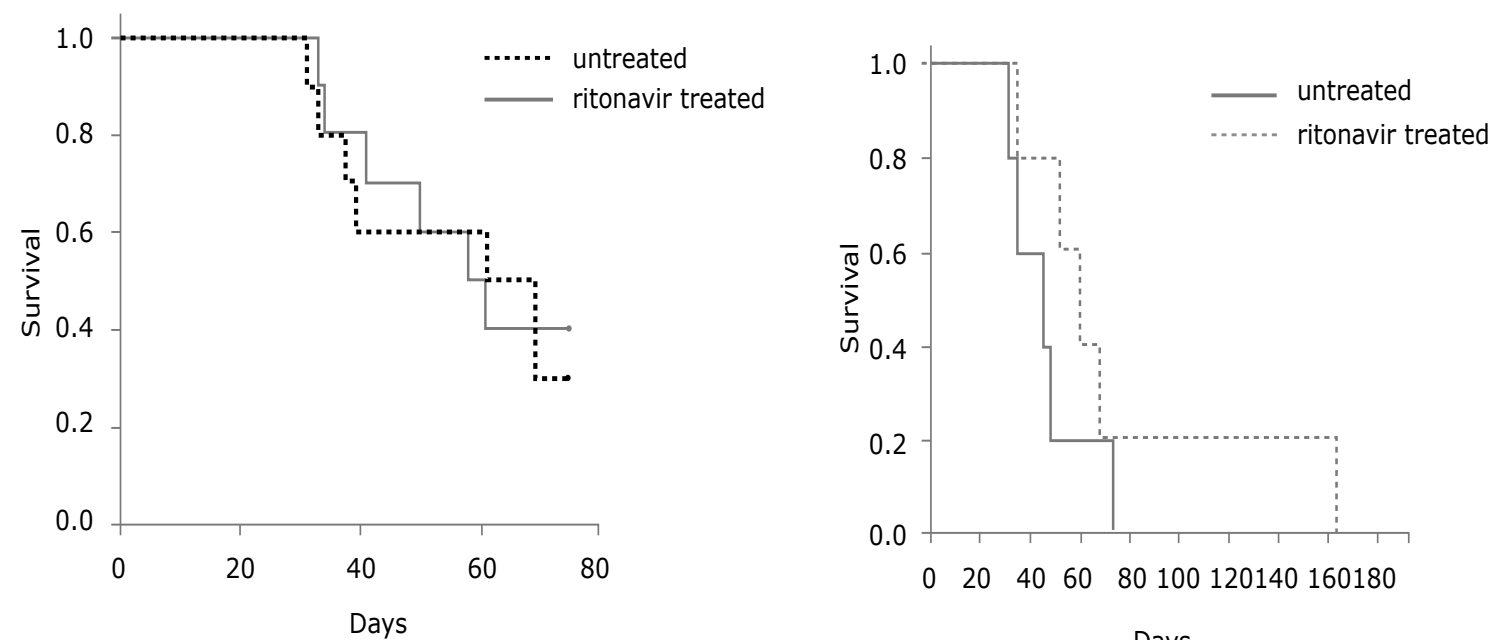

Figure 4: Efficacy of ritonavir as a single agent for treatment of MCL in vivo. Left Panel: Kaplan Meier Log Rank Test of efficacy with 7 days consecutive daily ritonavir treatment regimen in vivo in NOD/SCID mice inoculated with GP. Right Panel: Kaplan Meier Log Rank Test of efficacy with 30 days consecutive daily ritonavir treatment regimen in vivo in NOD/SCID mice inoculated with GP.
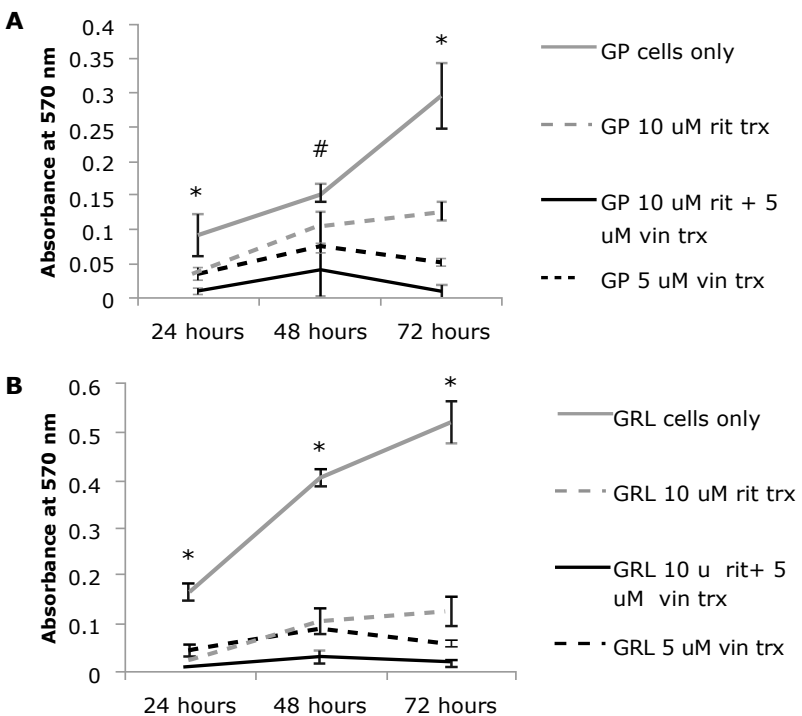

C
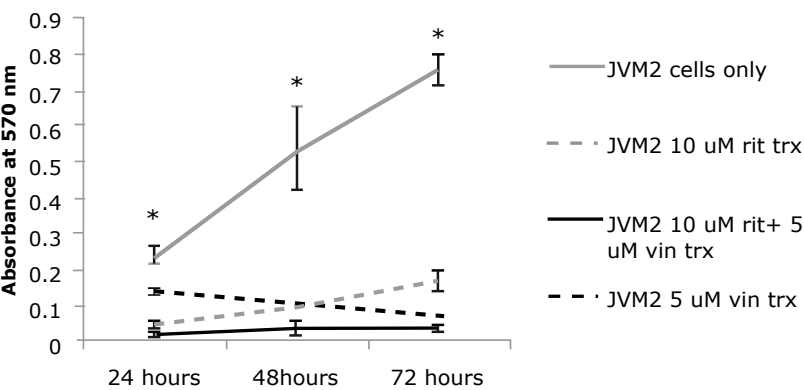

Figure 5: Efficacy of combined ritonavir + vincristine treatment in MCL. Effects of $10 \mu \mathrm{M}$ ritonavir treatment and/or $5 \mu \mathrm{M}$ vincristine treatment on the proliferation of GP (panel A), GRL (panel B), and JVM2 (panel C) after 24,48 , or 72 hour incubation periods, as assessed using MTT assays. GP, GRL, and JVM2 cell lines were seeded at 10,000 cells per well in a 96 well plate and incubated with $10 \mu \mathrm{M}$ ritonavir and/or $5 \mu \mathrm{M}$ vincristine for 24 48 , or 72 hours. Two hours prior to the given time point, $5 \mathrm{mg} / \mathrm{ml}$ MTT was added to each well, and cells were harvested at time point using a detergentbased buffer. Proliferation of the cells was assessed calorimetrically using a spectrophotometer. ${ }^{*} p<0.001$; \# $p=0.027$ comparing vincristine treatment alone to combined treatment with ritonavir and vincristine. measures for treating this disease. As scientific progress leads to the investigation of drug mechanisms and interactions, the potentials for alternate uses of FDA-approved pharmaceuticals in disparate disease settings has come to light. For example, while the therapeutic monoclonal antibody to $\mathrm{CD} 20$, rituximab, revolutionized the treatment of B-cell malignancies upon its FDA approval, rituximab is now wellappreciated and utilized in autoimmune/inflammatory conditions such as rheumatoid arthritis or systemic lupus erythematosus [32].

Another group of pharmaceuticals that has recently been studied for alternate uses is protease inhibitors. While originally developed as inhibitors of the HIV protease, this class of molecules is now being appreciated for its potential use in anti-cancer treatment regimens [33]. For example, the protease inhibitor ritonavir has demonstrated effects in various cancer types, including breast, prostate, and several hematological malignancies [14,18-20]. Therefore, improved therapy options for patients with MCL may be found in revisiting the varied potentials of FDA-approved drugs such as these protease inhibitors.

The results reported in this study suggest ritonavir may have only limited utility in treating MCL as a single agent. In vitro studies found that incubating MCL cell lines (GP, JVM2, and GP-derived therapyresistant GRL) with a concentration of ritonavir equivalent to serum levels in patients on daily ritonavir regimens significantly reduced cellular proliferation while inducing apoptosis. This data corroborates with previous reports that have shown reduced proliferation and increased apoptosis following ritonavir treatment in other hematological malignancies [14,20,34].

Reports suggesting the anti-cancer mechanisms of ritonavir may be through an alteration of signaling in the NFאB signaling pathways $[14,18,19]$. Therefore, transcript levels of the NFKB targets BCL2, CCND2, and BIRC5 were assessed following ritonavir treatment. These molecules were of particular interest due to their known roles in modulating survival and proliferation in MCL, amongst other cancers. In fact, disregulation of the BCL2 and survivin molecules has been shown to play a role in the progression, resistance, and/or aggressiveness of MCL, while cyclin D2 appears to serve as a functional replacement for cyclin D1 in rare MCL cases [35-39]. When treated for 6 hours with ritonavir, the transcript levels of BCL2 and CCND2 
in the GP, GRL, and JVM2 cell lines were significantly downregulated compared to untreated MCL cells. Interestingly, the BIRC5 transcript was also downregulated significantly in GP and GRL cells, but not in JVM2. This suggests intrinsic signaling mechanisms between the cell lines may be altering the molecular outcomes of ritonavir treatment amongst the cells, although all cell lines exhibited similar apoptosis induction and reduced proliferative outcomes.

In addition to assessing transcript level changes of NFkB pathway targets, the protein levels and expression patterns of $\mathrm{p} 65$, a subunit of $\mathrm{NF \kappa B}$, were analyzed in the GP, GRL, and JVM2 cell lines. Although there was no change in the total protein levels of p65 following 24 hours ritonavir treatment in the cells, there appeared to be a modest downregulation of $\mathrm{p} 65$ nuclear localization in the ritonavir-treated GP and GRL cells. This suggests ritonavir may be inhibiting some activation of NFKB. This modest decrease in nuclear localization suggests ritonavir action is not likely mediated solely through interaction with the NFKB signaling pathway, implicating a role for ritonavir actions in other signaling networks. Ritonavir interaction has been suggested to occur further upstream within the PI3K/Akt signaling pathway [19], providing insight into alternate pathways for consideration in determining ritonavir's mechanistic actions in MCL.

Due to the promising in vitro activity of ritonavir against MCL, we chose to test ritonavir's action in vivo. Two in vivo studies were performed to preliminarily test the efficacy of ritonavir as a single agent in treating MCL. In one dosing scheme, $30 \mathrm{mg} / \mathrm{kg} /$ day ritonavir was administered to mice for 7 consecutive days beginning at 10 days following inoculation with GP. In a second dosing scheme, devised to more closely resemble those previously shown effective against lymphoblastoid B cells in vivo [14], mice were administered $30 \mathrm{mg} /$ $\mathrm{kg} /$ day ritonavir for 30 consecutive days beginning at 7 days following inoculation with GP. Neither of these dosing schemes testing ritonavir as a single agent yielded significant prolongation in survival of treated mice over sham controls.

While ritonavir as a single agent displays no in vivo efficacy against MCL, studies suggest that ritonavir may be most optimal as a therapeutic agent when combined with other therapies, leading to a multi-target treatment approach $[18,25,26]$. When the GP, GRL1, and JVM2 cell lines were incubated with ritonavir in combination with a standard chemotherapeutic, vincristine, cell proliferation was significantly reduced compared to ritonavir treatment or vincristine treatment alone. This provides strong support for the development and testing of a combined therapeutic regimen with ritonavir and standard MCL therapeutics for in vivo testing, particularly due to extensive evidence suggesting multi-pronged therapies are more optimal treatments for MCL than single therapy designs that target only one aspect of the cancer's biology [23,24].

In conclusion, this study provides evidence for only limited utility of ritonavir in treating MCL. While ritonavir appears to have no in vivo activity against MCL as a single agent, evidence derived from in vitro studies holds promise for possible therapeutic potential of ritonavir utilization in a combined therapeutic regimen for treatment of MCL. Due to ritonavir's status as an FDA-approved drug, these studies warrant future in vivo studies for testing ritonavir in combined treatment regimens for more optimized MCL therapies. As MCL is an aggressive disease with a grim prognosis, these studies are vital to providing novel therapies better suited to managing MCL disease, thereby providing patients more optimal care and improved progression-free survival.

\section{Acknowledgements}

Financial support was provided through the Lymphoma Research Foundation, New York, NY.

\section{References}

1. Martinez A, Bellosillo B, Bosch F, Ferrer A, Marcé S, et al. (2004) Nuclear survivin expression in mantle cell lymphoma is associated with cell proliferation and survival. Am J Pathol 164: 501-510.

2. Zhou Y, Zhang L, Romaguera J, Delasalle K, Han X, et al. (2007) Immunotherapy in mantle cell lymphoma: anti-CD20-based therapy and beyond. Am J Hematol 83: 144-149.

3. Bertoni F, Rinaldi A, Zucca E, Cavalli F (2006) Update on the molecular biology of mantle cell lymphoma. Hematol Oncol 24: 22-27.

4. Fang Y, Vilella-Bach M, Bachmann R, Flanigan A, Chen J (2001) Phosphatidic acid-mediated mitogenic activation of mTOR signaling. Science 294: $1942-$ 1945

5. Semba S, Trapasso F, Fabbri M, McCorkell KA, Volinia S, et al. (2006) Fhit modulation of the Akt-survivin pathway in lung cancer cells: Fhit-tyrosine 114 (Y114) is essential. Oncogene 25: 2860-2872.

6. LoPiccolo J, Granville CA, Gills JJ, Dennis PA (2007) Targeting Akt in cancer therapy. AntiCancer Drugs 18: 861-874

7. Jares P, Colomer D, Campo E (2007) Genetic and molecular pathogenesis of mantle cell lymphoma: perspectives for new targeted therapeutics. Nat Rev Cancer 7: 750-762

8. Cecconi D, Zamo A, Bianchi E, Parisi A, Barbi S, et al. (2008) Signal transduction pathways of mantle cell lymphoma: a phosphoproteome-based study. Proteomics 8: 4495-4506.

9. Dal Col J, Zancai P, Terrin L, Guidoboni M, Ponzoni M, et al. (2008) Distinct functional significance of Akt and mTOR constitutive activation in mantle cel lymphoma. Blood 111: 5142-5151.

10. Shin S, Sung BJ, Cho YS, Kim HJ, Ha NC, et al. (2001) An anti-apoptotic protein human survivin is a direct inhibitor of caspase-3 and -7 . Biochemistry 40: 1117-1123.

11. Hansen JB, Fisker N, Westergaard M, Kjaerulff LS, Hansen HF, et al. (2008) SPC3042: a proapoptotic survivin inhibitor. Mol Cancer Ther 7: 2736-2745.

12. Minematsu T, Iwai M, Sugimoto K, Shirai N, Nakahara T, et al. (2009) Carrier-mediated uptake of 1-(2-methoxyethyl)-2-methyl-4,9-dioxo-3-(pyrazin2-ylmethyl)-4,9-dihydro-1H-naphtho[2,3-d]imidazolium bromide (YM155 monobromide), a novel small-molecule survivin suppressant, into human solid tumor and lymphoma cells. Drug Metab Dispos 37: 619-628.

13. Rodel F, Frey B, Leitmann W, Capalbo G, Weiss C, et al. (2008) Survivin antisense oligonucleotides effectively radiosensitize colorectal cancer cells in both tissue culture and murine xenograft models. Int J Radiat Oncol Biol Phys 71: 247-255

14. Dewan MZ, Tomita M, Katano H, Yamamoto N, Ahmed S, et al. (2009) An HIV protease inhibitor, ritonavir targets the nuclear factor-kappaB and inhibits the tumor growth and infiltration of EBV-positive lymphoblastoid B cells. Int $J$ Cancer 124: 622-629.

15. Carrasco RA, Stamm NB, Marcusson E, Sandusky G, Iversen P, et al. (2011) Antisense inhibition of survivin expression as a cancer therapeutic. Mol Cancer Ther 10: 221-232.

16. Tracey L, Pérez-Rosado A, Artiga MJ, Camacho FI, Rodríguez A, et al. (2005) Expression of the NF-kappaB targets BCL2 and BIRC5/survivin characterizes small B-cell and aggressive B-cell lymphomas, respectively. J Pathol 206: 123 134

17. Klier M, Anastasov N, Hermann A, Meindl T, Angermeier D, et al. (2008) Specific lentiviral shRNA-mediated knockdown of cyclin D1 in mantle cell lymphoma has minimal effects on cell survival and reveals a regulatory circuit with cyclin D2. Leukemia 22: 2097-2105.

18. Ikezoe T, Hisatake Y, Takeuchi T, Ohtsuki Y, Yang Y, et al. (2004) HIV1 protease inhibitor, ritonavir: a potent inhibitor of CYP3A4, enhanced the 
Citation: Nordgren TM, Hegde GV, Joshi SS (2012) Ritonavir Exhibits Limited Efficacy as a Single Agent in Treating Aggressive Mantle Cell Lymphoma. J Cancer Sci Ther 4: 061-068. doi:10.4172/1948-5956.1000112

anticancer effects of docetaxel in androgen-independent prostate cancer cells in vitro and in vivo. Cancer Res 64: 7426-7431.

19. Srirangam A, Mitra R, Wang M, Gorski JC, Badve S, et al. (2006) Effects of HIV protease inhibitor ritonavir on Akt-regulated cell proliferation in breast cancer. Clin Cancer Res 12: 1883-1896.

20. Dewan MZ, Uchihara JN, Terashima K, Honda M, Sata T, et al. (2006) Efficient intervention of growth and infiltration of primary adult T-cell leukemia cells by an HIV protease inhibitor, ritonavir. Blood 107: 716-724

21. Srirangam A, Milani M, Mitra R, Guo Z, Rodriguez M, et al. (2011) The human immunodeficiency virus protease inhibitor ritonavir inhibits lung cancer cells, in part, by inhibition of survivin. J Thorac Oncol 6: 661-670.

22. Ansell SM, Arendt BK, Grote DM, Jelinek DF, Novak AJ, et al. (2004) Inhibition of survivin expression suppresses the growth of aggressive non-Hodgkin's lymphoma. Leukemia 18: 616-623.

23. Dreyling M, Hiddemann W, European MCL Network (2009) Current treatment standards and emerging strategies in mantle cell lymphoma. Hematology Am Soc Hematol Educ Program 542-551.

24. Kahl BS (2009) Frontline therapy in mantle cell lymphoma: the role of high-dose therapy and integration of new agents. Curr Hematol Malig Rep 4: 213-217.

25. Zeldin RK, Petruschke RA (2004) Pharmacological and therapeutic properties of ritonavir-boosted protease inhibitor therapy in HIV-infected patients. J Antimicrob Chemother 53: 4-9.

26. Varma R, Carroll SL, Jacobson IV, Milross CG, Post JJ (2011) Combination antiretroviral therapy as treatment for human immunodeficiency virusassociated mucosa-associated lymphoid tissue type lymphoma of the nasopharynx. Intern Med J 41: 437-438.

27. Kraus M, Malenke E, Gogel J, Müller H, Rückrich T, et al. (2008) Ritonavir induces endoplasmic reticulum stress and sensitizes sarcoma cells toward bortezomib-induced apoptosis. Mol Cancer Ther 7: 1940-1948.

28. Oostendorp RL, Huitema A, Rosing H, Jansen RS, Ter Heine R, et al. (2009) Coadministration of ritonavir strongly enhances the apparent oral bioavailability of docetaxel in patients with solid tumors. Clin Cancer Res 15: 4228-4233.

29. Hegde GV, Nordgren TM, Munger CM, Mittal A, Bierman P, et al. (2012) Novel therapy for therapy-resistant mantle cell lymphoma: multipronged approach with targeting hedgehog signaling. Int $\mathrm{J}$ Cancer.

30. Hegde GV, Munger CM, Emanuel K, Joshi AD, Greiner TC, et al. (2008) Targeting of sonic hedgehog-GLI signaling: a potential strategy to improve therapy for mantle cell lymphoma. Mol Cancer Ther 7: 1450-1460.

31. Geisler $\mathrm{CH}$ (2010) Front-line treatment of mantle cell lymphoma. Haematologica 95: $1241-1243$

32. McDonald V, Leandro M (2009) Rituximab in non-haematological disorders of adults and its mode of action. Br J Haematol 146: 233-246.

33. Bernstein WB and Dennis PA (2008) Repositioning HIV protease inhibitors as cancer therapeutics. Curr Opin HIV AIDS 3: 666-675

34. Gaedicke S, Firat-Geier E, Constantiniu O, Lucchiari-Hartz M, Freudenberg M et al. (2002) Antitumor effect of the human immunodeficiency virus protease inhibitor ritonavir: induction of tumor-cell apoptosis associated with perturbation of proteasomal proteolysis. Cancer Res 62: 6901-6908.

35. O'Connor DS, Grossman D, Plescia J, Li F, Zhang H, et al. (2000) Regulation of apoptosis at cell division by p34cdc2 phosphorylation of survivin. Proc Nat Acad Sci U S A 97: 13103-13107.

36. Quintanilla-Martinez L, Slotta-Huspenina J, Koch I, Klier M, Hsi ED, et al. (2009) Differential diagnosis of cyclin D2+ mantle cell lymphoma based on fluorescence in situ hybridization and quantitative real-time-PCR. Haematologica 94: 15951598.

37. Gesk S, Klapper W, Martin-Subero JI, Nagel I, Harder L, et al. (2006) A chromosomal translocation in cyclin D1-negative/cyclin D2-positive mantle cell lymphoma fuses the CCND2 gene to the IGK locus. Blood 108: 1109-1110.

38. Bogner C, Dechow T, Ringshausen I, Wagner M, Oelsner M, et al. (2010) Immunotoxin BL22 induces apoptosis in mantle cell lymphoma (MCL) cells dependent on Bcl-2 expression. Br J Haematol 148: 99-109.

39. Metcalf RA, Zhao S, Anderson MW, Lu ZS, Galperin I, et al. (2010) Characterization of D-cyclin proteins in hematolymphoid neoplasms: lack of specificity of cyclin-D2 and D3 expression in lymphoma subtypes. Mod Patho 23: $420-433$. 